\title{
DESCRIPCIÓN SINCRÓNICA DEL ÁREA PALATAL DE HABLANTES NATIVOS DE RESISTENCIA, ARGENTINA
}

\section{Synchronic description to palatal area of native speakers in the city of Resistencia, Argentina}

\author{
Hugo Roberto Wingeyer* \\ Universidad Nacional del Nordeste \\ hugowingeyer@gmail.com
}

\author{
Olga Natalia Trevisán** \\ Universidad Nacional del Nordeste \\ ontrevisan@gmail.com
}

\section{Palabras clave región NEA de Argentina; área palatal; proceso de cambio; deslateralización}

\begin{abstract}
RESUMEN
El contraste entre los fonemas palatal sonoro fricativo central, $/ \mathrm{j} /, \mathrm{y}$ palatal sonoro lateral, $/ \lambda /$, caracterizado como una oposición aislada y de escaso rendimiento funcional, es un rasgo fonológico propio del español de la región NEA de Argentina. Los procesos de cambio del área palatal han sido investigados en estudios cuantitativos y observaciones participativas. En este trabajo identificamos la situación actual de los hablantes nativos de Resistencia, ciudad capital de la provincia del Chaco. Para eso, presentamos un estudio cuantitativo, elaborado con un corpus de lengua oral, formado por entrevistas realizadas entre 2013 y 2018 sistematizado de acuerdo con factores extralingüísticos (nivel educativo, edad y sexo), en el que observamos un avance significativo del proceso de deslaterialización.
\end{abstract}

\begin{abstract}
The contrast between the central voiced palatal fricative, $/ \mathrm{j} /$, and the voiced palatal lateral approximant, $/ \lambda /$, phonemes, characterised as an isolated and of poor functional utility opposition, is a phonological feature of Spanish in the Argentina NEA region. The change processes of the palatal area have been studied in quantitative researches and participatory observations. In this paper we identify the current situation of native speakers in Resistencia, the capital city of Chaco province. For that purpose, we present a quantitative study of a spoken corpus, comprised by interviews carried out between 2013 and 2018, sorted according to extra-linguistic factors (educational level, age and gender), in which we observe a significant progress in the delaterialization process.
\end{abstract}

\section{Keywords}

Argentina NEA region; process of change; palatal area; de-lateralization 


\section{Introducción}

El objetivo de este trabajo es la identificación del estado de situación actual de un fenómeno del área palatal de los nativos de la ciudad de Resistencia, capital de una de las cuatro provincias que integran la región nordeste de Argentina (NEA): Misiones, Corrientes, Chaco y Formosa. Nos referimos al grado de avance del proceso de deslateralización de $/ K /$, el yeísmo, y su caracterización en el español sincrónico de dicha ciudad.

Si bien el proceso de desfonologización de la oposición fonema central / fonema palatal a favor del primero, es un fenómeno ampliamente extendido en España y América (Peña Arce, 2015; Gómez y Molina Martos, 2013; Aleza Izquierdo, 2010; Quilis y Quilis-Sanz, 2002; Vaquero, 1996; Lipski, 1994; Canfield, 1981, 1988), esta oposición se mantiene en Paraguay (de Granda 1980, 1982, 1988; Alvar, 1996), Bolivia (Quilis y Quilis Sanz, 2003; Gordon, 1980), Perú (Calvo Pérez, 2008) y en las zonas nordeste (de influjo guaraní) y noroeste (próxima a la cordillera andina) de Argentina.

En lo que respecta a Paraguay, determinados estudios demuestran que, contrariamente a la mayoría de Hispanoamérica, se mantiene la oposición fonológica en estudio "casi en su totalidad", hasta el punto de considerar la articulación lateral un "emblema y motivo de orgullo para los paraguayos” (Peña Arce, 2015, p. 194). A su vez, la articulación de la /j/, reforzada por el contacto español-guaraní, ${ }^{1}$ es africada en todas las posiciones. En los sociolectos urbanos medios y altos, es alveolar y ligeramente ensordecida, sobre todo en mujeres. Se ha señalado asimismo rehilamiento de /j/ tras -s (Aleza Izquierdo, 2010, p.69).

La región NEA de Argentina, estrechamente relacionada con Paraguay, por razones geográficas, de evolución histórica y demográfica, es una área usualmente caracterizada como distinguidora de la oposición $/ \mathrm{j} /-/ \mathrm{K} /$, cuenta con estudios previos sobre el registro de procesos de cambio en el área palatal de hablantes nativos de sus ciudades capitales: sobre Corrientes, Abadía de Quant (1988, 1996, 2003) y Wingeyer y Trevisán (2013), sobre Formosa y Posadas, Wingeyer y Trevisán (2018a y 2018b, respectivamente).

Respecto de Resistencia, Abadía de Quant $(1996,2004)$ considera que "los procesos de cambio registran (en esta ciudad) el mayor avance observado en la región” (2004, p. 128). Afirma que la causa fundamental de la pérdida de $/ K /$ y el avance de la deslateralización ocasional es la "incorporación de hablantes sureños de isolectos medio y alto que emigraron hacia la ciudad desde fines de la década del cincuenta atraídos por sus requerimientos económicos y culturales” (p. 128).

1. Si bien de Granda reconoce la influencia del guaraní, considera que este refuerzo se debe a una causación múltiple (1980, 1982, 1988). Ya en su artículo Factores determinantes de la preservación del fonema /ll en el español del Paraguay de 1979 señaló la presencia de vascos durante la época de formación y consolidación del español como factor que reforzó el mantenimiento de la lateral en Paraguay. 


\section{Sobre la ciudad en estudio}

Resistencia es la capital de la provincia del Chaco, su ciudad más poblada y centro social y económico de la provincia. Es, a su vez, la cabecera del Departamento San Fernando y del área metropolitana, conocida como Gran Resistencia. Ubicada al sudeste de la provincia, sobre la orilla derecha del río Paraná, se encuentra a $18 \mathrm{~km}$ de la ciudad de Corrientes, con la que forma un conurbano de cerca de 800000 habitantes.

La ciudad, fundada en 1878 por Decreto del Gobierno Nacional sobre la base del Paraje San Fernando, asentamiento habitado desde mediados del siglo XIX, recibió un contingente de inmigrantes italianos que formaron la primera colonia agrícola del Chaco, que permitió luego la colonización del resto del territorio (Maeder, 1996, p. 129-130). En 1884 fue declarada Capital del Territorio Nacional del Chaco y en las décadas posteriores se transformó en la urbe más poblada del NEA, favorecida por las buenas vías de comunicación que le otorgan el río Paraná y las vías del ferrocarril (Maeder, 1996, p. 130). La habilitación del del Puente General Belgrano en 1973, la potenció como nudo comunicacional del Norte Argentino, conectándola con la ciudad de Corrientes y con el resto de la Mesopotamia argentina.

La población de Resistencia está formada por descendientes de inmigrantes europeos, criollos venidos de provincias vecinas y el Paraguay, y descendientes de pueblos originarios. Entre los criollos la mayor parte proviene de la vecina provincia de Corrientes; los correntinos y paraguayos formaban la mayor parte de la población antes de la creación de la colonia. Entre los pueblos originarios tiene mayor preponderancia la etnia qom, aunque también hay wichís y mocovíes.

Hacia fines del siglo XX el movimiento migratorio de la población rural y de localidades del interior de la provincia hacia Resistencia provocaron un fuerte aumento de su población. Luego, en el periodo intercensal 2001-2010, ${ }^{2}$ la población del Chaco se incrementó un 7,1\% y en el Departamento San Fernando, la variación, en igual periodo fue de 6.6\%. Específicamente en el municipio de Resistencia la variación en el periodo intercensal fue de un 5,3\%.

En cuanto a su caracterización lingüística, por su situación geográfica, su formación histórica y evolución demográfica, Resistencia pertenece a la región nordeste (Donni de Mirande, 1992, p. 384-400), área de influencia de la lengua guaraní, caracterizada principalmente por la retención de modalidades tradicionales y por las influencias morfosintácticas y fonético-fonológicas del guaraní en el español.

2. Obtuvimos los datos estadísticos del portal Estadísticas Chaco http://estadisticas.chaco.gob.ar/censos/ y procesados a través del programa CEPAL REDATAM. 


\section{Sobre la muestra}

Tomando en cuenta los antecedentes del tema en estudio, en este trabajo analizamos un corpus de lengua oral, formado por entrevistas realizadas entre 2013 y 2018 a noventa y seis informantes hablantes nativos de la ciudad de Resistencia.

Para la recolección de datos utilizamos varios tipos de estrategias, en un total aproximado de treinta horas de grabación. En un primer momento, los investigadores, hablantes nativos de la variedad del español en estudio, recurrimos tanto a interacciones sociales participativas como al papel de meros observadores en situaciones naturales de conversación libre. Luego, para poder examinar la pronunciación de nuestros informantes en contextos formales, les solicitamos la lectura en voz alta de un texto informativo breve.

La muestra, cuyo tamaño es el adecuado para la aproximación al fenómeno en estudio, fue organizada acorde con los siguientes factores extralingüísticos contextualizadores: nivel educativo (instrucción primaria, completa o incompleta, secundaria y universitaria), edad (cuatro grupos de ente 18 y 60 años) y sexo. Se trata, en consecuencia, de un grupo de informantes heterogéneo respecto de las variables consideradas. En cuanto al número de hablantes, mantuvimos la misma cantidad de participantes en todos los casos (Cuadro 1).

\begin{tabular}{|l|c|c|c|c|c|c|c|c|c|c|c|c|c|}
\hline (Cuadro 1) & \multicolumn{10}{|c|}{ Número de hablantes por edad, sexo y nivel de instrucción } \\
\hline & \multicolumn{3}{|c|}{$18-30$} & \multicolumn{3}{c|}{$31-40$} & \multicolumn{3}{c|}{$41-50$} & \multicolumn{3}{c|}{$51-60$} & \\
\hline & $\mathrm{P}$ & $\mathrm{S}$ & $\mathrm{U}$ & $\mathrm{P}$ & $\mathrm{S}$ & $\mathrm{U}$ & $\mathrm{P}$ & $\mathrm{S}$ & $\mathrm{U}$ & $\mathrm{P}$ & $\mathrm{S}$ & $\mathrm{U}$ & \\
\hline \multirow{2}{*}{ RESISTENCIA } & Mujer & 4 & 4 & 4 & 4 & 4 & 4 & 4 & 4 & 4 & 4 & 4 & 4 \\
\cline { 2 - 16 } & Varón & 4 & 4 & 4 & 4 & 4 & 4 & 4 & 4 & 4 & 4 & 4 & 4 \\
\hline
\end{tabular}

Fuente: Elaboración propia a partir de entrevistas grabadas en las cuatro ciudades capitales de la región NEA

\section{Análisis de la muestra}

\subsection{Consideraciones generales}

\subsubsection{Observaciones sobre el procesamiento de las entrevistas}

Tanto las grabaciones de las interacciones y lecturas, como su análisis auditivo, fueron realizados por nosotros, los autores de este trabajo. Asimismo, consideramos que los hablantes que completaron el proceso de deslateralización, en los que no registramos en ningún caso la realización del fonema palatal lateral $/ \mathrm{K} /$, son los que completaron el proceso de deslateralización. Por último, aclaramos que la deslateralización ocasional es la fluctuación en la utilización del fonema palatal lateral y su reemplazo por variantes fricativas y africadas del fonema central. 


\subsubsection{El tamaño relativo de la muestra}

Para la determinación del tamaño relativo de la muestra, compuesta por noventa y seis informantes, tomamos los datos demográficos relativos a la ciudad de Resistencia del programa CEPAL REDATAM, correspondiente al censo 2010. En cuanto a los datos de la composición de la población, los habitantes de Resistencia, nacidos en la provincia del Chaco, suman 243923; por consiguiente, nuestra muestra, de noventa y seis individuos nativos de la ciudad capital, constituye un 0,039\% de ese total. Ahora bien, si consideramos solo a los hablantes de más de 18 años, es decir, a aquellos sobre los que se realizó la unidad primaria de observación, el total de informantes se reduce a 160923, lo que representa el 0,059\% de ese total (Cuadro 2).

\begin{tabular}{|l|c|c|c|}
\hline (Cuadro 2) & Total de hablantes nativos & $\begin{array}{l}\text { Total de hablantes nativos mayores de } \\
18 \text { años }\end{array}$ & $\begin{array}{l}\text { Porcentaje representativo } \\
\text { de la muestra }\end{array}$ \\
\hline Resistencia & 243923 & 160400 & $0.059 \%$ \\
\hline
\end{tabular}

Fuente: Elaboración propia a partir del programa CEPAL REDATAM correspondiente al censo 2010

Un dato estadístico de interés para nuestra investigación, aportado por el mismo censo 2010, es el de números de personas provenientes de Paraguay en la composición de la población extranjera de la ciudad en estudio (Cuadro 3).

\begin{tabular}{|l|c|c|}
\hline (Cuadro 3) & Total de inmigrantes & Inmigrantes de nacionalidad paraguaya \\
\hline Resistencia & 3180 & 1856 \\
\hline
\end{tabular}

Fuente: Elaboración propia a partir del programa CEPAL REDATAM correspondiente al censo 2010

\subsubsection{Unidades de análisis}

En lo que respecta a la oposición fonológica en estudio: fonema palatal sonoro fricativo central y fonema palatal sonoro lateral $(/ \mathrm{j} /-/ \lambda /)$, en las transcripciones de nuestra investigación, hallamos las siguientes unidades: ${ }^{3}$

$/ \lambda /$ fonema palatal fricativo sonoro lateral

[3] variante palatal fricativa sonora central

3. A diferencia de lo que ocurre en las otras ciudades capitales de la región NEA de Argentina, no registramos la variante

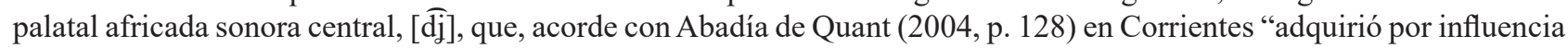
del guaraní el rasgo de africación”. 
[d] variante palatal africada rehilada central

$\left[\int\right]$ variante palatal fricativa sorda. ${ }^{4}$

\subsection{Análisis del objeto de estudio de acuerdo con las variables consideradas}

\subsubsection{Presentación de resultados generales}

Sobre la base de la distribución planteada en el Cuadro 1, presentamos ahora un cuadro por cada grupo de hablantes de acuerdo con la variable edad (hablantes nativos de entre 51-60 años o más, hablantes nativos de entre 41-50 años, hablantes nativos de entre 31-40 años y hablantes nativos de entre 18-30 años). Además, consideramos en cada uno de estos cuadros las variables sexo y nivel de instrucción. Finalmente, observamos que cada una de las referencias incluidas en las distintas celdas, a partir de entrevistas grabadas en cada una de las ciudades en estudio, representa a un informante.

En el grupo de informantes de 51 a 60 años o más, no registramos el fenómeno de la desfonologización de la oposición fonema lateral-fonema central, a favor de este último. En lo que respecta al fonema central, solamente aparecen variantes rehiladas, fricativas y africadas.

Por último, no observamos diferencias significativas respecto de la variable sexo (Cuadro 4).

\begin{tabular}{|c|c|c|c|c|c|c|}
\hline \multirow[t]{3}{*}{ (Cuadro 4) } & \multicolumn{6}{|c|}{ Hablantes nativos entre 51-60 o más años } \\
\hline & \multicolumn{2}{|c|}{$\begin{array}{l}\text { Nivel primario completo/in- } \\
\text { completo }\end{array}$} & \multicolumn{2}{|c|}{$\begin{array}{l}\text { Nivel secundario completo/ } \\
\text { incompleto }\end{array}$} & \multicolumn{2}{|c|}{$\begin{array}{l}\text { Nivel universitario completo/ } \\
\text { incompleto }\end{array}$} \\
\hline & Mujer & Varón & Mujer & Varón & Mujer & Varón \\
\hline \multirow[t]{4}{*}{ Resistencia } & $/ \lambda /-[d]$ & $/ \lambda /-[d]$ & $/ \lambda /-[3 \sim d \xi]$ & $/ \lambda /-[3 \sim d z]$ & $/ \lambda /-[3 \sim d z]$ & $/ \lambda /-\left[3 \sim d_{3}\right]$ \\
\hline & $/ \lambda /-[3 \sim d\}]$ & $/ \lambda /-[3 \sim d \zeta]$ & $/ \lambda /-[3 \sim d z]$ & $/ \lambda /-[3 \sim d z]$ & $/ \lambda /-[3 \sim d\}]$ & $/ \lambda /-[3]$ \\
\hline & $/ \lambda /-[3 \sim d\}]$ & $/ \lambda /-[3 \sim d\}]$ & $/ \lambda /-[3]$ & $/ \lambda /-[3 \sim d\}]$ & $/ \lambda /-[3]$ & $/ \lambda /-[3]$ \\
\hline & $/ \lambda /-[3 \sim d z]$ & $/ \lambda /-[3 \sim d \xi]$ & $/ \lambda /-\left[d s \sim \int\right]$ & $/ \lambda /-[3]$ & $/ \lambda /-\left[\mathrm{d} J \sim \int\right]$ & $/ \lambda /-[3]$ \\
\hline
\end{tabular}

Cuadro 4. Distribución de fonemas y variantes en el primer conjunto de hablantes (51-60 o más años) de acuerdo con las variables sexo y nivel de instrucción

En los hablantes nativos del conjunto de 41 a 50 años (Cuadro 5) observamos deslateralización en seis de los ocho informantes de nivel universitario y en cuatro de nivel secundario; lo que nos indica el avance del proceso en esta ciudad. Finalmente, en este grupo etario tampoco observamos diferencias significativas respecto de la variable sexo.

\footnotetext{
4. Para explicar por qué en el yeísmo del español de Buenos Aires el fonema /j/ es sustituido por las unidades fricativas [3] y [S], fenómeno que desde allí se irradia a otras provincias argentinas, Abadía de Quant recurre a la estrecha convivencia con portugueses desde el siglo XVII hasta la primera mitad del siglo XIX (2008, p.188). Sostiene que "el gallego, vasco, francés, italiano sobre todo dialectal sureño, e inglés, lenguas que en el área palatal tenían unidades sordas, o sorda y sonora(s) rehilada(s) cuando en el período colonial y siglo XIX, estuvieron en contacto con el español de Buenos Aires, con variado grado de intensidad y simultaneidad, habrían podido apoyar el proceso de cambio iniciado por el portugués como desencadenante primario". (Abadía de Quant, 2008, p.191)
} 


\begin{tabular}{|c|c|c|c|c|c|c|}
\hline \multirow[t]{3}{*}{ (Cuadro 5) } & \multicolumn{6}{|c|}{ Hablantes nativos entre $41-50$ años } \\
\hline & \multicolumn{2}{|c|}{$\begin{array}{l}\text { Nivel primario completo/ } \\
\text { incompleto }\end{array}$} & \multicolumn{2}{|c|}{$\begin{array}{l}\text { Nivel secundario completo/ } \\
\text { incompleto }\end{array}$} & \multicolumn{2}{|c|}{$\begin{array}{l}\text { Nivel universitario completo/ } \\
\text { incompleto }\end{array}$} \\
\hline & Mujer & Varón & Mujer & Varón & Mujer & Varón \\
\hline \multirow[t]{4}{*}{ Resistencia } & $\left./ \lambda / \mathrm{d}_{3}\right]$ & $/ \lambda /{ }^{\prime}[\mathrm{d}]$ & $/ \lambda /\left[3 \sim d_{3}\right]$ & $/ \lambda /-\left[3 \sim d_{3}\right]$ & $/ \lambda /-[3]$ & $\mid \lambda /-\left[3 \sim d_{3}\right]$ \\
\hline & $/ \lambda /-\left[3 \sim d_{3}\right]$ & $/ \lambda /-\left[3 \sim d_{3}\right]$ & $/ \lambda /-[3]$ & $/ \lambda /-[3 \sim d z]$ & $\varnothing-\left[3 \sim d_{3}\right]$ & $\varnothing-[3 \sim d z]$ \\
\hline & $/ \lambda /-\left[3 \sim d_{3}\right]$ & $/ \lambda /-\left[3 \sim d_{3}\right]$ & $\varnothing-[3 \sim d z]$ & $\varnothing-[3 \sim d 3]$ & $\varnothing-\left[3 \sim d_{3}\right]$ & $\varnothing-[3 \sim d z]$ \\
\hline & $/ \lambda /-[3]$ & $/ \lambda /-[3]$ & $\varnothing-[3 \sim d z]$ & $\varnothing-[3 \sim d]$ & $\varnothing-[3]$ & $\varnothing-[3]$ \\
\hline
\end{tabular}

Cuadro 5. Distribución de fonemas y variantes en el primer conjunto de hablantes (41-50 años) de acuerdo con las variables sexo y nivel de instrucción

En el conjunto de 31 a 40 años (Cuadro 6) registramos casos de desfonologización en la totalidad de informantes universitarios y en cuatro secundarios. Por consiguiente, destacamos, en el avance del fenómeno en estudio, que el primer conjunto de informantes ya completó el proceso de cambio al no registrarse el uso del fonema palatal lateral en ninguna instancia del intercambio verbal. Por otro lado, si bien se registran variantes fricativas y africadas del fonema central rehilado, es importante el avance del fonema central fricativo. Por último, seguimos sin observar diferencias significativas respecto de la variable sexo en todo el grupo etario.

\begin{tabular}{|c|c|c|c|c|c|c|}
\hline \multirow[t]{3}{*}{ (Cuadro 6) } & \multicolumn{6}{|c|}{ Hablantes nativos entre $31-40$ años } \\
\hline & \multicolumn{2}{|c|}{$\begin{array}{l}\text { Nivel primario completo/ } \\
\text { incompleto }\end{array}$} & \multicolumn{2}{|c|}{$\begin{array}{l}\text { Nivel secundario completo/ } \\
\text { incompleto }\end{array}$} & \multicolumn{2}{|c|}{$\begin{array}{l}\text { Nivel universitario completo/ } \\
\text { incompleto }\end{array}$} \\
\hline & Mujer & Varón & Mujer & Varón & Mujer & Varón \\
\hline \multirow[t]{4}{*}{ Resistencia } & $/ \lambda /-[3 \sim d \xi]$ & $\lambda /-[3 \sim \mathrm{d} 3]$ & $/ \lambda /-[3 \sim d 3]$ & $/ \lambda /-[3]^{*}$ & $\varnothing-[3 \sim d]$ & $\varnothing-[3 \sim d z]$ \\
\hline & $/ \lambda /-[3 \sim d\}]$ & $/ \lambda /-[3 \sim \mathrm{d} 3]$ & $/ \lambda /-[3 \sim d 3]^{*}$ & $/ \lambda /-[3]^{*}$ & $\varnothing-[3 \sim d b]$ & $\varnothing-[3 \sim d z]$ \\
\hline & $/ \lambda /-[3]^{*}$ & $/ \lambda /-[3]$ & $\varnothing-[3]$ & $\varnothing-[3 \sim d 3]$ & $\varnothing-[3]$ & $\varnothing-[3]$ \\
\hline & $/ \lambda /-[3]^{*}$ & $/ \lambda /-[3]^{*}$ & $\varnothing-[3]$ & $\varnothing-\left[3 \sim \int\right]$ & $\varnothing-[3]$ & $\varnothing-[3]$ \\
\hline
\end{tabular}

Cuadro 6. Distribución de fonemas y variantes en el primer conjunto de hablantes (31-40 años) de acuerdo con las variables sexo y nivel de instrucción

En el conjunto de menor edad, observamos que completan el proceso de desfonologización cinco universitarios de Resistencia y seis de nivel secundario (Cuadro 7). A su vez, registramos varios casos de desfonologización ocasional. Además, en lo que respecta al fonema central, se registran variantes rehiladas fricativas y africadas, y se observa que continúa el avance del fonema central fricativo. Finalmente, tampoco aquí encontramos diferencias significativas respecto de la variable sexo. 


\begin{tabular}{|c|c|c|c|c|c|c|}
\hline \multirow[t]{3}{*}{ (Cuadro 7) } & \multicolumn{6}{|c|}{ Hablantes nativos entre 18-30 años } \\
\hline & \multicolumn{2}{|c|}{$\begin{array}{l}\text { Nivel primario completo/ } \\
\text { incompleto }\end{array}$} & \multicolumn{2}{|c|}{$\begin{array}{l}\text { Nivel secundario completo/ } \\
\text { incompleto }\end{array}$} & \multicolumn{2}{|c|}{$\begin{array}{l}\text { Nivel universitario completo/ } \\
\text { incompleto }\end{array}$} \\
\hline & Mujer & Varón & Mujer & Varón & Mujer & Varón \\
\hline \multirow[t]{4}{*}{ Resistencia } & $\mid \lambda /-[3 \sim d z]^{*}$ & $/ \lambda /-[3 \sim d z]$ & $\mid \lambda /-[3 \sim d z]^{*}$ & $/ \lambda /-[3 \sim d\}]^{*}$ & $/ \lambda /-[3 \sim \mathrm{d} b]^{*}$ & $/ N /[3 \sim d z]^{*}$ \\
\hline & $\mid \lambda /-\left[3 \sim \mathrm{d}_{3}\right]^{*}$ & $/ \lambda /-[3 \sim d z]^{*}$ & $\varnothing-\left[3 \sim d_{3}\right]$ & $\varnothing-[3 \sim d 3]$ & $\mid \lambda /-\left[3 \sim \mathrm{d}_{3}\right]^{*}$ & $\varnothing-\left[\mathrm{d} \sim 3 \sim \int\right]$ \\
\hline & $\varnothing-[3 \sim d\}]$ & $\varnothing-[3 \sim d z]$ & $\varnothing-[3 \sim d\}]$ & $\varnothing-[3 \sim d 3]$ & $\varnothing-[d]$ & $\varnothing-[3]$ \\
\hline & $\varnothing-[3 \sim d]$ & $\varnothing-[3 \sim d z]$ & $\varnothing-\left[3 \sim d_{3}\right]$ & $\varnothing-\left[3 \sim d_{3}\right]$ & $\varnothing-[3]$ & $\varnothing-[3]$ \\
\hline
\end{tabular}

Cuadro 7. Distribución de fonemas y variantes en el primer conjunto de hablantes (18-30 años) de acuerdo con las variables sexo y nivel de instrucción

Presentamos ahora el conjunto de datos considerando todas las variables lingüísticas contextualizadoras:

1. Registramos casos de desfonologización de la oposición fonema lateral-fonema central, por parte del 63\% de los informantes universitarios y del 75\% de los informantes con estudios secundarios del grupo de 18-30 años. Además, observamos, en el mismo grupo etario, casos de desfonologización ocasional en el $37 \%$ de los estudiantes universitarios y en el $25 \%$ de los informantes de nivel secundario.

Verificamos el mismo fenómeno de desfonologización en la totalidad de los informantes universitarios y en el 50\% de los informantes de nivel secundario del grupo de 31 a 40; grupo en el que se observa desfonologización ocasional en el 38\% de los informantes de nivel secundario.

En el grupo de 41-50 años, encontramos casos de desfonologización en el 100\% de los informantes universitarios y en $50 \%$ de los informantes de nivel secundario. También se registran casos de desfonologización ocasional en tres de los informantes de nivel secundario.

2. En lo que respecta al fonema palatal central, aparece en todos los conjuntos la alternancia de las variantes rehiladas fricativa [3] y africada [d] , y a partir del conjunto de 41-50 años se verifica el avance del fonema central fricativo rehilado.

3. Registramos la variante sorda ${ }^{5}$ solo en cuatro oportunidades: en dos informantes de 50-60 años o más, una mujer con instrucción secundaria y otra, universitaria, en un informante de nivel secundario de 31-40 años, y en uno de entre 18-30 años con instrucción universitaria.

4. En general, no se observan diferencias significativas respecto de la variable sexo.

5. Observamos que si bien en la primera investigación sobre los procesos de cambio en el área palatal de los nativos de la ciudad de Corrientes, Abadía de Quant (1988) no registró esta variante sorda a la que se consideraba "valorada como estereotipo lingüístico del habla de los nativos de Buenos Aires" (p. 24), aparece un caso en el corpus de la investigación realizada posteriormente en la misma ciudad por Wingeyer Trevisan. (2013, p. 99) 


\subsubsection{Resultados generales según las variables grupo etario y nivel de instrucción}

En los dos gráficos que mostramos seguidamente se representan los resultados de acuerdo con las variables en las que se observan diferencias significativas: grupo etario y nivel de instrucción. En el Gráfico 1 se ven los fonemas y sus variantes según el grupo etario. Las líneas de tendencia, seleccionadas en función del registro del mayor número de casos del mantenimiento de la oposición palatal central - palatal lateral $(/ K /-[3 \sim d \xi])$ y del proceso de deslateralización $(\varnothing-[3 \sim$ d $]$ ] $)$, dan cuenta del avance de esta última tendencia en los hablantes de menor edad.

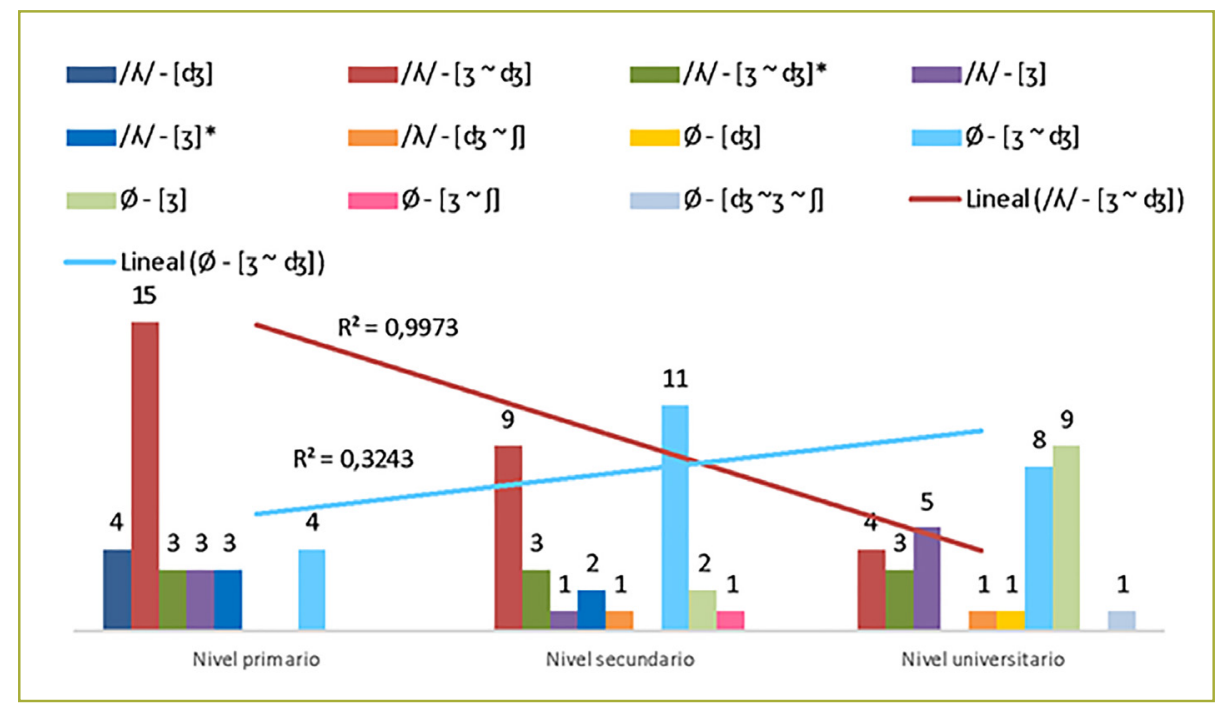

Gráfico 1. Distribución de fonemas y variantes de acuerdo con la variable edad

Por otro lado, en el Gráfico 2, en el que se toma en cuenta la variable nivel de instrucción, vemos que las líneas de tendencia, seleccionadas, como en el Gráfico 1, en función del mayor número de registros, muestran el avance del proceso de deslateralización en los informantes con mayor nivel de instrucción, en tanto que la oposición de los fonemas palatal central - palatal lateral se mantiene entre los hablantes con menor nivel de instrucción.

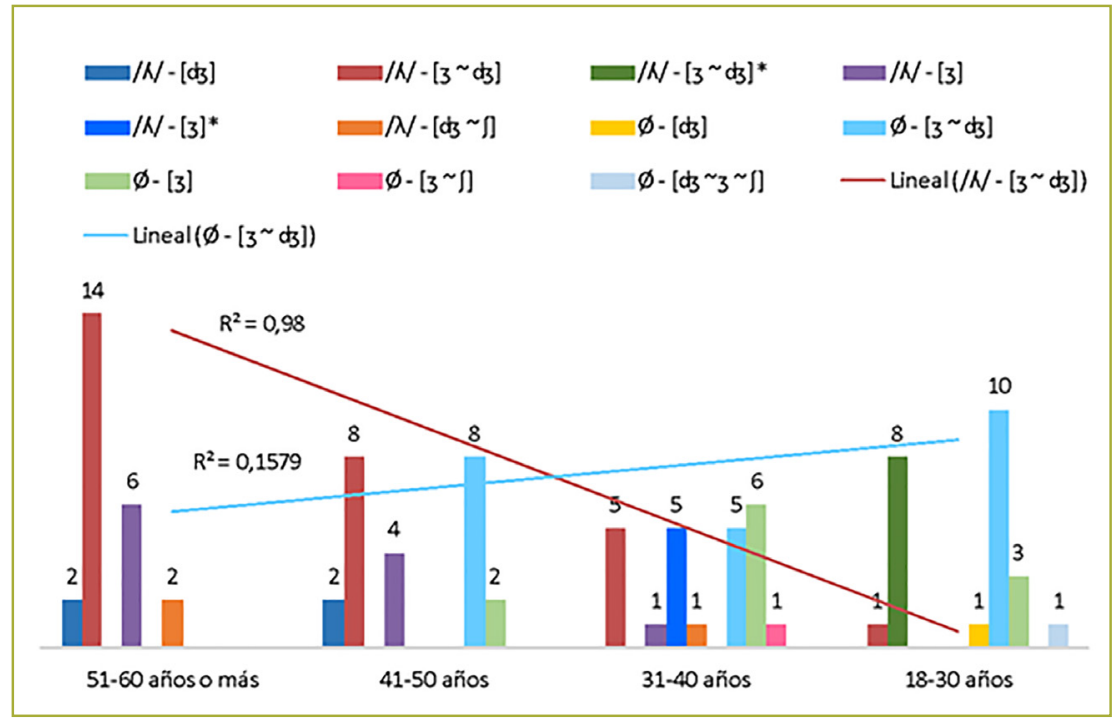

Gráfico 2. Distribución de fonemas y variantes de acuerdo con la variable nivel de instrucción 


\subsubsection{Observaciones del avance de la desfonologización considerando las variables grupo etario, nivel de instrucción y sexo}

Según la hipótesis de partida, el avance del fenómeno de deslateralización en los hablantes nativos de la ciudad en estudio puede apreciarse con mayor claridad en los tres gráficos porcentuales en los que se toman en cuenta las variables consideradas en esta investigación: nivel de instrucción, grupo etario y sexo. Se observa que los que mayoritariamente completaron el proceso de desfonologización son los informantes con estudios secundarios y universitarios de 18-30.

En el Gráfico 3, registramos casos de desfonologización en el 12.50\% del grupo de nivel primario, se incrementa en el conjunto de instrucción secundaria, donde alcanza el 43,75\%, y llega al 59\% en el nivel universitario. A esta información agregamos el significativo avance de la desfonologización ocasional entre el nivel primario y el secundario. Desde otro punto de vista, se advierte el mantenimiento de la oposición palatal central-palatal lateral en el 87.50\% de los hablantes de nivel primario.

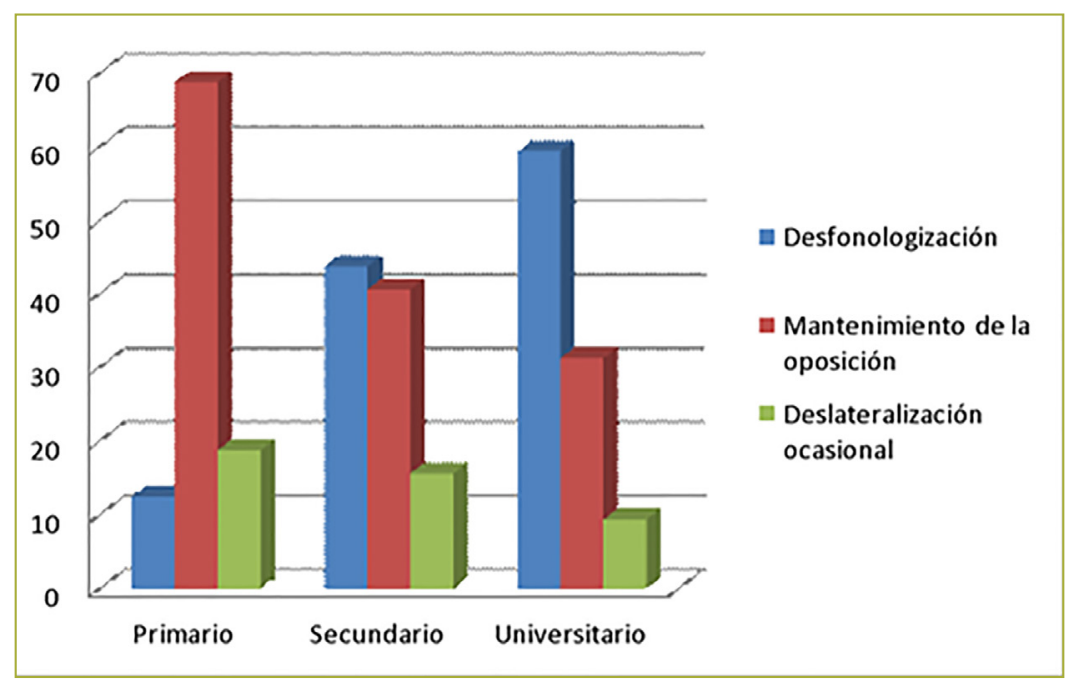

Gráfico 3. Distribución porcentual del proceso de desfonologización de acuerdo con la variable nivel de instrucción

En el Gráfico 4, el avance más significativo se evidencia en el conjunto de menor edad, el de 18-30 años, en el que el 62.50\% de nuestros informantes completó el proceso de deslateralización. Observamos que el 100\% \% de los hablantes de mayor edad mantiene la oposición palatal central / palatal lateral, que decrece en forma progresiva al disminuir la edad, hasta llegar al 37.50\% en el grupo de 18-30 años. 


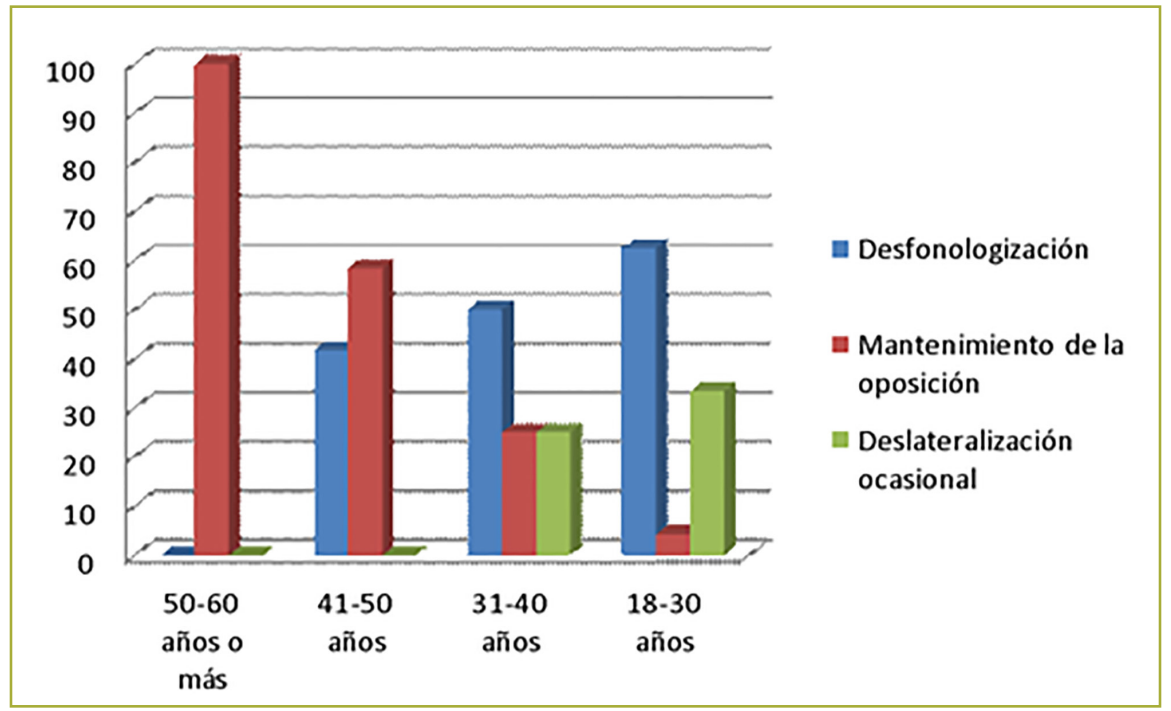

Gráfico 4. Distribución porcentual del proceso de desfonologización de acuerdo con la variable edad

Observamos en el Gráfico 5, como ya ha sido considerado, que no hay diferencias significativas entre mujeres y varones en lo que respecta al mantenimiento de la oposición de los fonemas palatal central - palatal lateral y su proceso de desfonologización.

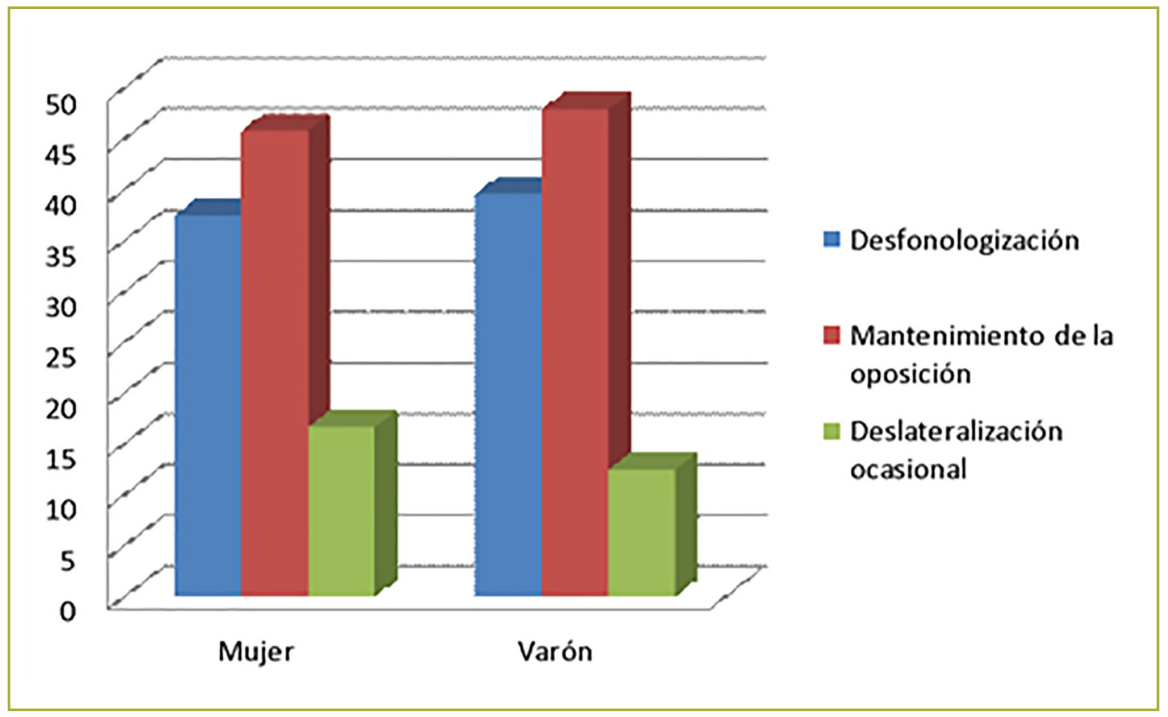

Gráfico 5. Distribución porcentual del mantenimiento de la oposición palatal lateral-palatal central y su desfonologización de acuerdo con la variable sexo 


\subsection{Análisis cuantitativo: la prueba de chi-cuadrado $\left(\chi^{2}\right)$. Efectos de las variables sexo, nivel de instrucción y grupo etario en la variable dependiente grado de desfonologización}

Para asegurarnos de que la asociación entre las variables es significativa, y que no es azarosa, recurrimos el test estadístico de significación chi-cuadrado $\left(\chi^{2}\right) \cdot{ }^{6}$ En nuestro caso, en lo que respecta a la variable independiente sexo, observamos que no hay una asociación estadística significativa entre esta variable y el proceso de desfonologización: $(\chi 2=0.335, \mathrm{df}=2, \mathrm{p}=0.846)$. En el caso de la variable nivel de instrucción, detectamos una asociación estadística significativa entre esta variable independiente y el proceso de desfonologización; es decir que cuanto más alto es el nivel, mayor es el porcentaje de desfonologización: $(\chi 2=15.7$, df $=4, \mathrm{p}<0.05)$. Finalmente, en lo que concierne a la variable independiente edad, también registramos una asociación estadística significativa entre esta variable y el proceso de desfonologización: $(\chi 2=55.2, \mathrm{df}=6, \mathrm{p}<0.05)$.

\subsection{Ejemplificación del avance del fonema central fricativo rehilado y constatación del registro de una variante sorda sobre la base de su análisis acústico}

Para empezar, presentamos la información recogida de los ocho informantes del conjunto de menor edad y de mayor nivel de instrucción, sobre los procesos de desfonologización y transfonologización:

1. E1 37,50\% de los informantes de menor edad desfonologiza ocasionalmente y el 62,50\% ya completó el proceso de desfonologización. A su vez, el el 37,50\% de estos últimos hablantes completó también el proceso de transfonologización a favor de la variante central fricativa.

2. Solo en un informante registramos en toda posición en la palabra la variante central africada.

6. Esta prueba compara la frecuencia esperada de las variables con la frecuencia observada. Si el valor de chi calculado es menor que el chi de la tabla, se acepta la hipótesis, puesto que el chi calculado se encuentra dentro del intervalo de confianza. 


\section{Aparece en un informante una variante sorda.}

Exponemos ahora, de manera inicial y exploratoria, el análisis acústico ${ }^{7}$ de dos informantes de este grupo de hablantes nativos de Resistencia. Lo hacemos con el propósito de ilustrar el avance del fonema central fricativo rehilado en el proceso de transfonologización en estudio y constatar la presencia de una variante sorda que, si bien no es de uso tan frecuente en el área, está presente en los hablantes de menor edad y mayor nivel de instrucción.

En el análisis acústico del informante A (Figura 1), una joven de 21 años con estudios universitarios incompletos, observamos el proceso de transfonologización, con la presencia de la variante rehilada en posición intervocálica; en el caso del informante B (Figura 2), joven de 19 con estudios universitarios incompletos, identificamos la variante sorda. Distinguimos en los espectrogramas la energía aleatoria con apariencia de lluvia para las fricativas sordas y de lluvia mezclada con estriaciones característica de los pulsos glotales para las sonoras. Vemos también en la barra de sonoridad en bajas frecuencias.

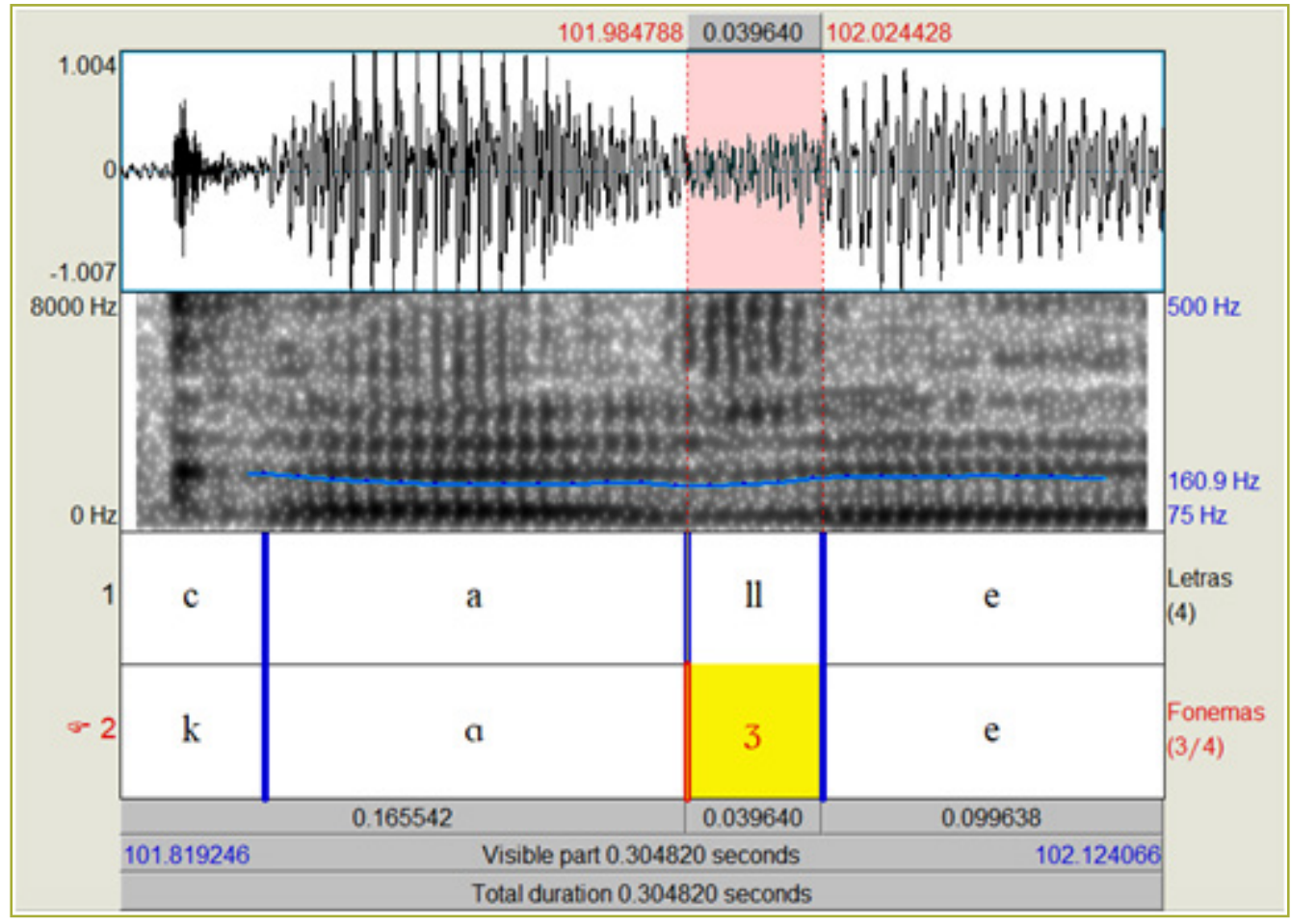

Figura 1. Hablante A. Visualización del espectrograma de la variante palatal fricativa rehilada central en la palabra calle

7. Observamos que para la obtención de los espectrogramas, en el programa Praat, ajustamos la frecuencia entre 0 y 8.000 $\mathrm{Hz}$. 


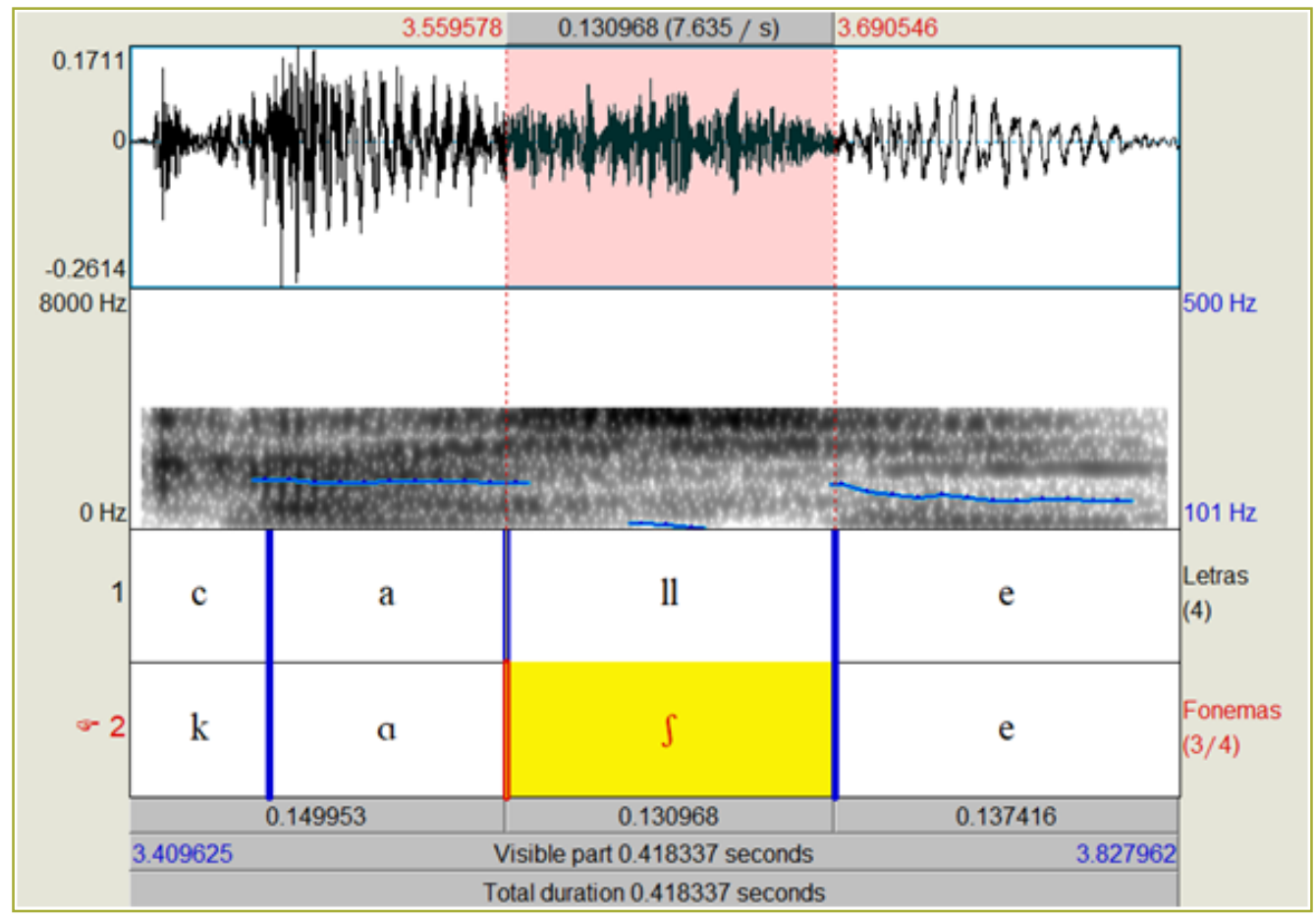

Figura 2. Hablante B. Visualización del espectrograma de la variante palatal fricativa sorda en la palabra calle

\section{Conclusiones}

Tal como planteamos en la introducción, cumplimos con nuestro propósito de aportar información sobre el fenómeno del yeísmo en la ciudad de Resistencia. una de las ciudades capitales de las provincias que integran la región NEA de Argentina, tradicionalmente considerada como área distinguidora de la oposición fonema central - fonema palatal. Hemos registrado en nuestros informantes avances muy significativos en el proceso de cambio del área palatal de sus hablantes nativos, proceso en el que destacamos la observación en nuestros informantes de fenómenos de deslateralización, tanto en los que están en proceso de cambio como en los que ya lo han cumplido, a favor de un fonema central; nos referimos a los informantes secundarios y universitarios de 18-50 años. Además, observamos en todos los grupos la fluctuación de variantes africadas y fricativas del rehilado central, con un mayor avance en el proceso de transfonologización a favor de estas últimas en el grupo de menor edad y mayor nivel de instrucción.

Finalmente, observamos que, acorde con los resultados de la aplicación de la prueba de chi-cuadrado $\left(\chi^{2}\right)$, se detectan asociaciones estadísticas significativas entre las variables independientes nivel de instrucción y edad, y el proceso de desfonologización. Asimismo, el análisis acústico nos permite, entre los hablantes de 18-30 años con estudios secundarios y universitarios completos o incompletos, ilustrar el avance de la variante fricativa rehilada y constatar la presencia de una variante sorda.

\section{Referencias bibliográficas}

Aleza Izquiero, M. (2010). Oposición entre palatales / Yeísmo y rehilamiento. En Aleza Izquierdo, M 
y Enguita Utrilla, J. M. (coords.), La lengua española en América: normas y usos actuales (pp. 68-70). Valencia, Universitat de Valencia.

Abadía de Quant, I. (1988). Procesos de cambios en el área palatal de los nativos de Corrientes, Argentina. Anuario de Lingüistica Hispánica, V, 9-25.

Abadía de Quant, I. (1996). Sistemas lingüísticos en contacto y sus consecuencias en el área palatal del español de dos capitales del Nordeste argentino: Corrientes y Resistencia. International Journal of The Sociology of Language, 11-25.

Abadía de Quant, I. (2003). Aspectos del español coloquial sincrónico de los nativos de la capital de Corrientes (Argentina). Resistencia, Universidad Nacional del Nordeste.

Abadía de Quant, I. (2004). El español del Nordeste. En Fontanella de Weinberg, M. (ed.), El español de la Argentina y sus variedades regionales (pp. 121-160). Bahía Blanca, Asociación Bernardino Rivadavia.

Abadía de Quant, I. (2008). El sistema fonológico portugués, desencadenante temprano de un proceso de cambio en el español porteño. En Mailhe, A. y Reitano, E. (comps.), Pensar Portugal: Reflexiones sobre el legado histórico y cultural del mundo luso en Sudamérica (pp. 165-193). [en línea]. La Plata, Universidad Nacional de La Plata. https://bit.ly/32bwqy9.

Alvar, M. (dir.) (1996). Manual de dialectología hispánica (vol. II: El español de América). Barcelona, Ariel.

Canfield, D. (1981). Spanish Pronunciation in the Americas. Chicago, University of Chicago Press.

Donni de Mirande, N. (1992). El español actual hablado en la Argentina. En Hernández Alonso, C. (ed.), Historia y Presente del Español de América (pp. 383-410). Valladolid, PABECAL.

Gómez, R. y Molina Martos, I. (eds.) (2013). Variación yeista en el mundo hispánico. Madrid, VervuertIberoamericana.

Gordon, A. M. (1980). Notas sobre la fonética del castellano en Bolivia. Actas del Sexto Congreso Internacional de Hispanistas, 349-352.

Granda, G. de (1979). Factores determinantes de la preservación del fonema /11/ en el español del Paraguay. Lingüistica Española Actual, 1, 403-412.

Granda, G. de (1980). Algunos rasgos fonéticos del español paraguayo atribuibles a interferencia guaraní. Revista Española de Lingüistica, 10, 339-349.

Granda, G. de (1982). Observaciones sobre la fonética del español del Paraguay. Anuario de Letras, 20, 145-194.

Granda, G. de (1988). Observaciones sobre la fonética del español del Paraguay. En Sociedad, historia y lengua en el Paraguay (pp. 109-156). Bogotá, Instituto Caro y Cuervo.

Lipski, J. (1994). Latin American Spanish. Londres y Nueva York, Longman. 
Maeder, E. (1996). Historia del Chaco. Buenos Aires, Editorial Plus Ultra.

Peña Arce, J. (2015). Yeísmo en el español de América. Algunos apuntes sobre su extensión. Revista de Filología, 33, 175-199.

Quilis, A. y Qulis Sanz, M.J. (2002). El español en América. Madrid, UNED.

Quilis, A.y Qulis Sanz, M.J. (2003). Datos para la caracterización fonética del español de Bolivia. En Moreno, F. et alii (eds.), Lengua, variación y contexto. Estudios dedicados a Humberto López Morales (pp. 775-791). Madrid, Arco.

Vaquero de Ramírez, M. (1996). El español de América (vol. I: Pronunciación). Madrid, Arco Libros.

Wingeyer, H. R. y Trevisán, O. N. (2013). Procesos de cambio del área palatal de hablantes nativos de la ciudad de Corrientes, Argentina. Contextos, 30, 93-100.

Wingeyer, H. R. y Trevisán, O. N. (2018a). Descripción sincrónica del área palatal de hablantes nativos de Formosa, Argentina. Cuadernos de la Alfal, 10, 192-204.

Wingeyer, H. R. y Trevisán, O. N. (2018b). Descripción sincrónica del área palatal de hablantes nativos de Posadas, Argentina. Rasal 2018, 87-104.

*Hugo Roberto Wingeyer es Doctor por la Universidad de Alcalá (Programa de Lingüística Aplicada) y se desempeña como Profesor Titular de Historia del Español y del Taller de Comprensión y Producción de Textos de la Facultad (Universidad Nacional del Nordeste). Dirige el Proyecto de Investigación: "Descripción y análisis de prácticas letradas académicas de estudiantes de la Facultad de Humanidades de la UNNE 2017/2020” (SGCyT-UNNE).

** Olga Natalia Trevisán es Profesora en Letras por la Universidad Nacional del Nordeste y Magíster en Enseñanza de la Lengua y la Literatura por la Universidad Nacional de Rosario. Se desempeña como Profesora Adjunta en Lengua y cultura latinas y como Auxiliar en Historia del español en la Facultad de Humanidades (UNNE). Actualmente desarrolla su tesis doctoral titulada "Modalidades de reescritura de las obras clásicas grecolatinas destinadas a niños y jóvenes de Argentina (1940-2019)”, en el marco del Doctorado en Ciencias Humanas y Sociales de la Universidad Nacional de Misiones.

RECIBIDO: $20 / 11 / 2020$

Aceptado: 20/12/2020 